\title{
A FACTOR THEOREM FOR FRECHET MANIFOLDS
}

BY R. D. ANDERSON AND R. SCHORI ${ }^{1}$

Communicated by R. D. Anderson, August 9, 1968

1. Introduction. A Frechet manifold (or F-manifold) is a separable metric space $M$ having an open cover of sets each homeomorphic to an open subset of the countable infinite product of open intervals, $s$. A $Q$-manifold is a separable metric space $M$ having an open cover of sets each homeomorphic to an open subset of the Hilbert cube, $I^{\infty}$. It is known that all separable metric Banach manifolds modeled on separable infinite-dimensional Banach spaces are $F$-manifolds. The following are the principle theorems of this paper.

THEOREM I. If $M$ is any F-manifold, then $s \times M$ is homeomorphic to $M$.

THEOREM II. If $M$ is any $Q$-manifold, then $I^{\infty} \times M$ is homeomorphic to $M$.

Since $s$ is known, [1] or [3], to be homeomorphic to $s \times I^{\infty}$, from Theorem I we immediately have the following.

COROLlaRy. If $M$ is any F-manifold, then $I^{\infty} \times M$ is homeomorphic to $M$.

Almost identical proofs of Theorems I and II can be given. To emphasize the ideas of our proofs of Theorems I and II we shall outline instead a proof of the similar but notationally easier

ThEOREM I'. If $M$ is any F-manifold and $J^{0}$ is the open interval $(-1,1)$, then $J^{0} \times M$ is homeomorphic to $M$.

2. Lemma 2.1 implies Theorem I'.

Definition. Let $r$ be a map, i.e. continuous function, of a space $X$ into the closed unit interval $[0,1]$. Let $J^{0}(0)=\{0\}$ and for $t \in(0,1]$, let $J^{0}(t)=(-t, t)$. Then $J^{0} X^{r} X=\left\{(y, x) \in J^{0} \times X: y \in J^{0}(r(x))\right\}$ is the variable product of $J^{0}$ by $X$ (with respect to $r$ ).

Lemma 2.1. Let $U$ be an open subset of $s$, let $V \subset W \subset U$ where $W$ is open and $V$ is closed in $U$, and let $J^{0} \times^{\text {to }} U$ be a variable product of $J^{0}$

1 This paper is a brief resume of a paper Factors of infinite-dimensional manifolds, submitted by the authors to Trans. Amer. Math. Soc. The research was supported in part under NSF grants GP 6867 and GP 8637. 
by $U$. There exists a homeomorphism $H$ of $J^{0} X^{r 0} U$ onto a variable product $J^{0} \times^{r} U$ such that (1) $r \leqq r_{0}$, (2) $r(V)=0$, and (3) $H \mid J^{0} \times^{r 0}\left[(U \backslash W) \cup r_{0}^{-1}(0)\right]$ is the identity.

OUtline of a PRoof that Lemma 2.1 implies Theorem I'. Since $M$ is separable and metric, there exists a countable star-finite open cover $G$ of $M$ with sets homeomorphic to open subsets of $s$. (By starfinite cover we mean a cover such that the closure of each element intersects only finitely many closures of other members of the cover.) Thus if $V \subset U$ where $U \in G$ and $V$ is closed in $M$ and $J^{0} \times^{r 0} M$ is a variable product of $J^{0}$ by $M$, then by taking an open set $W$ in $M$ such that $W \supset V$ and $\mathrm{Cl}_{M} W \subset U$, Lemma 2.1 will imply the existence of a homeomorphism of $J^{0} X^{r_{0}} U$ that has an automatic extension to a homeomorphism $H$ of $J^{0} \times^{r_{0}} M$ where (1) $r \leqq r_{0}$, (2) $r(V)=0$, and (3) $H \mid J^{0} X^{r_{0}}\left[(M \backslash U) \cup r_{0}^{-1}(0)\right]$ is the identity.

As suggested by Theorem 2 of [2], we take a special ordering of the elements of $G$, say $\left\{U_{i}\right\}_{i>0}$. Now take a cover $\left\{V_{i}\right\}_{i>0}$ of $M$ where, for each $i>0, V_{i}$ is a closed set contained in $U_{i}$. For each $i>0$, let $H_{i}$ be a homeomorphism from $J^{0} \times^{r_{i-1}} M$ onto $J^{0} \times^{r_{i}} M$ where $r_{i} \leqq r_{i-1} \leqq \cdots \leqq r_{0}=1, r_{i}\left(V_{i}\right)=0$, and $H_{i} \mid J^{0} \times^{r_{i-1}}\left(M \backslash U_{i}\right)$ is the identity. Then $\left(H_{i} \circ \cdots \circ H_{1}\right)_{i>0}$ converges to a homeomorphism of $J^{0} \times M$ onto $\{0\} \times M$ which is homeomorphic to $M$.

3. Two lemmas leading to Lemma 2.1. Let $s=\prod_{i>0} J_{i}^{0}$ where for each $i>0, J_{i}^{0}=J^{0}$. Let $\pi: J^{0} \times s \rightarrow s$ be the natural projection onto $s$ and for $n>0$, let $\pi_{n}$ be defined on $s$ as follows. For $z=\left(z_{1}, z_{2}, \cdots\right) \in s$, let $\pi_{n}(z)=\left(z_{1}, \cdots, z_{n}, 0,0, \cdots\right)$. Also, for $Y$ a space and $f: s \rightarrow Y$, define $f^{*}: J^{0} \times s \rightarrow Y$ by $f^{*}=f \pi$.

LEMmA 3.1. There exists a map

$$
h:\left(J^{0} \times s\right) \times[0,1] \times[1, \infty) \rightarrow J^{0} \times s
$$

such that if $t \in[0,1]$ and $u \in[1, \infty)$ are fixed where $n \leqq u$, the map

$$
H: J^{0} \times s \rightarrow J^{0} \times s
$$

defined by $H(p)=h(p, t, u)$ for $p \in J^{0} \times s$ is a homeomorphism of $J^{0} \times s$ onto $J^{0} X^{r} s$ where (1) $r=1-t$, (2) if $t=0, H$ is the identity, and (3) $\pi_{n}^{*}=\pi_{n}^{*} H$.

Outline of Proof. It suffices to describe $h$. We first describe for any integer $n>0$ a map $h^{\prime}$ of $\left(J^{0} \times s\right) \times[0,1) \times\{n\}$ onto $J^{0} \times s$.

For $x=\left(x_{0}, x_{1}, \cdots\right) \in J^{0} \times s$, let $h^{\prime}(x, 0, n)=x$ and as $t$ varies from 0 to $\frac{1}{2}$ let the 0 th and $(n+1)$ th coordinates of $x$ be "rotated" so that at $t=\frac{1}{2},\left(x_{0}, x_{n+1}\right)$ becomes $\left(x_{n+1},-x_{0}\right)$ while for $0 \leqq t \leqq \frac{1}{2}$ all other coordinates are left fixed. 
Thus, $h^{\prime}\left(x, \frac{1}{2}, n\right)=\left(x_{n+1}, x_{1}, \cdots, x_{n},-x_{0}, x_{n+2}, \cdots\right)$. For $i>0$, as $t$ varies from $1-2^{-i}$ to $1-2^{-i-1}$, "rotate" the 0 th and $(n+i+1)$ th coordinates leaving all other coordinates fixed so that for each $i>0$,

$h^{\prime}\left(x, 1-2^{-i}, n\right)$

$$
=\left(x_{n+i}, x_{1}, \cdots, x_{n},-x_{0},-x_{n+1}, \cdots,-x_{n+i-1}, x_{n+i+1}, \cdots\right) .
$$

To define $h$ from $h^{\prime}$ we specify that $h(x, 1, n)=\left(0, x_{1}, \cdots, x_{n},-x_{0}\right.$, $\left.-x_{n+1},-x_{n+2}, \cdots\right)$ and we introduce for any time $t<1$, a factor of $1-t$ in the 0th coordinate place of $h^{\prime}$. Indeed for each integer $n \geqq 1, h \mid\left(J^{0} \times s\right) \times[0,1] \times\{n\}$ becomes an isotopy. It is now possible to extend the domain of $h$ for values of $u$ between $n$ and $n+1$ by use of "rotations" similar in nature to the "rotations" used in defining $h^{\prime}$ for a fixed $n$.

An open set $E$ of $s$ is an $n$-basic open set in $s$ if $E=E_{1} \times \cdots \times E_{n}$ $\times \prod_{i>n} J_{i}^{0}$ where each $E_{i}$ is open in $J_{i}^{0}$ and is a subinterval of $J_{i}^{0}$.

Definition. Let $W$ be open in $s$ and let $\left\{G_{i}\right\}$ be a star finite collection of $m_{i}$-basic open sets in $s$ whose union is $W$. For each $x \in W$ let

$$
m_{x}=\operatorname{minimum}\left\{m_{i}: x \in G_{i}\right\} .
$$

Let $Y$ be a space. A map $f: W \rightarrow Y$ is a local product map of $W$ with respect to the $G_{i}$ and $m_{i}$ if $f(x)=f\left(\pi_{m_{x}}(x)\right)$ for each $x \in W$. If, additionally, $Y=[1, \infty)$ and $f(x) \geqq m_{x}$ for each $x \in W$, then $f$ is a local product indicator map of $W$ with respect to the $G_{i}$ and the $m_{i}$.

The strategy is to replace the $t$ and $u$ of Lemma 3.1 by local product maps. The following technical lemma (not proved here) asserts the existence of the proper type of local product maps.

LEMma 3.2. Let $U$ be an open subset of $s$ and let $V \subset W \subset U$ and $A \subset U$ where $W$ is open and $V$ and $A$ are closed in $U$. There exist a countable star finite collection $\left\{G_{i}\right\}$ of $m_{i}$-basic open sets in $s$ whose union is $W \backslash A$ and maps $\phi: U \backslash A \rightarrow[0,1]$ and $g: W \backslash A \rightarrow[1, \infty)$ such that (1) $\phi(\bigvee A)=1,(2) \phi((U \backslash W) \backslash A)=0$, (3) $\phi \mid W \backslash A$ and $g$ are $a$ local product map and a local product indicator map, respectively, of $W \backslash A$ with respect to the $G_{i}$ and $m_{i}$, and (4) $g$ is unbounded near $A$, that is, for any $x \in A \cap \mathrm{Cl}(W \backslash A)$ and $n>0$, there is a neighborhood $B(x)$ such that $g \mid(W \backslash A) \cap B(x)>n$.

4. Proof of Lemma 2.1. By Lemma 3.2 take a star finite collection $\left\{G_{i}\right\}$ of $m_{i}$-basic open sets and the maps $\phi$ and $g$ for the case when $A=r_{0}^{-1}(0)$. Now, let

$$
h:\left(J^{0} \times s\right) \times[0,1] \times[1, \infty) \rightarrow J^{0} \times s
$$


be the map of Lemma 3.1 and define

$$
H_{1}: J^{0} \times W \backslash r_{0}^{-1}(0) \rightarrow J^{0} \times s
$$

by $H_{1}(p)=h\left(p, \phi^{*}(p), g^{*}(p)\right)$ for $p \in J^{0} \times W \backslash r_{0}^{-1}(0)$. It can be shown that $H_{1}$ is a homeomorphism onto $J^{0} X^{r_{1}} W \backslash r_{0}^{-1}(0)$ where $r_{1}=1-\phi$. Clearly the map $k$ from $J^{0} \times^{r_{0}} W \backslash r_{0}^{-1}(0)$ to $J^{0} \times W \backslash r_{0}^{-1}(0)$ defined by $k(y, z)=\left(y r_{0}^{-1}(z), z\right)$ is a homeomorphism. Also $k^{-1} H_{1} k$ is a homeomorphism from $J^{0} \times^{r_{0}} W \backslash r_{0}^{-1}(0)$ onto $J^{0} \times^{r} W \backslash r_{0}^{-1}(0)$ where $r=(1-\phi) r_{0}$. Now define $H: J^{0} \times^{r_{0}} U \rightarrow J^{0} \times^{r} U$ by $H=k^{-1} H_{1} k$ on $J^{0} X^{r_{0}} W \backslash r_{0}^{-1}(0)$ and $H=$ identity on $J^{0} \times\left[(U \backslash W) \cup r_{0}^{-1}(0)\right]$. We show that $H$ is continuous. Since $\phi\left((U \backslash W) \backslash r_{0}^{-1}(0)\right)=0$, from condition 2 of Lemma 3.1 it follows that $H \mid J^{0} \times\left(W \backslash r_{0}^{-1}(0)\right)$ and the identity map on $J^{0} \times\left[(U \backslash W) \backslash r_{0}^{-1}(0)\right]$ are compatible. To show that these are compatible with the identity $J^{0} \times{ }^{r_{0}} r_{0}^{-1}(0)$ we check the coordinatewise continuity of $H$. The continuity of $r_{0}$ gives the continuity of $H$ on the first, or $J^{0}$, coordinate and $g$ becoming unbounded near $r_{0}^{-1}(0)$ yields the continuity of $H$ on the second, or $U$, coordinate. The other conditions of Lemma 2.1 are easily seen to be satisfied.

\section{REFERENCES}

1. R. D. Anderson, Topological properties of the Hilbert cube and the infinite product of open intervals, Trans. Amer. Math. Soc. 126 (1967), 200-216.

2. R. D. Anderson, David W. Henderson and James E. West, Negligible subsets of infinite-dimensional manifolds, Compositio Math. (to appear).

3. Czeslaw Bessaga and Victor Klee, Every non-normable Fréchet space is homeomorphic with all of its closed convex bodies, Math. Ann. 163 (1966), 161-166.

Louisiana State University, Baton Rouge, Louisiana 70803 